Notre Dame Journal of Formal Logic

Volume 36, Number 4, Fall 1994

\title{
On Finite-Valued Propositional Logical Calculi
}

\author{
O. ANSHAKOV and S. RYCHKOV
}

\begin{abstract}
In this paper we describe, in a purely algebraic language, truthcomplete finite-valued propositional logical calculi extending the classical Boolean calculus. We also give a new proof of the Completeness Theorem for such calculi. We investigate the quasi-varieties of algebras playing an analogous role in the theory of these finite-valued logics to the role played by the variety of Boolean algebras in classical logic.
\end{abstract}

1 Introduction Assume that an $n$-valued logic $L_{n}, n>1$, is given by means of truth tables. We shall denote the set of truth values by $V=\{0,1 /(n-1), \ldots,(n-$ $2) /(n-1), 1\}$. Assume MARK $\subseteq V$, where $1 \in$ MARK and $0 \notin$ MARK. We shall call MARK the collection of designated (marked) truth values. Let the algebra of the logic $L_{n}$ be $\left\langle V, \sigma_{1}\right\rangle$, where the signature $\sigma_{1}$ consists of operations on $V$. The logic $L_{n}$ is completely determined by the triple $\left\langle V, \sigma_{1}\right.$, MARK $\rangle$.

Small Greek letters $\alpha, \beta, \ldots$, shall be used to denote arbitrary truth values. We say that the logic $L_{n}$ is truth-complete iff all J-operators $\left\{\mathrm{J}_{\alpha}^{*} \mid \alpha \in V\right\}$ are functionally expressible in the signature $\sigma_{1}$, where (see Rosser 48])

$$
\mathrm{J}_{\alpha}^{*} \beta= \begin{cases}1 & \text { if } \beta=\alpha \\ 0 & \text { if } \beta \neq \alpha .\end{cases}
$$

Intuitively, the expressibility in the signature $\sigma_{1}$ of all J-operators $\left\{\mathrm{J}_{\alpha}^{*} \mid \alpha \in V\right\}$ means that, for every $\alpha \in V$, it is possible to say in the language of the logic $L_{n}$ that a proposition $A$ assumes the given truth value $\alpha$.

A logic $L_{n}$ is said to be $\mathbf{C}$-extending iff in $L_{n}$ one can functionally express the binary operations $\wedge^{*}, \vee^{*}, \supset^{*}$, and the unary operation $\neg^{*}$ (whose restrictions to the subset $\{0,1\}$ of $V$ coincide with the classical logical operations of conjunction, disjunction, implication, and negation). Note that $L_{n}$ coincides with classical logic $\mathbf{C}$ over the set $\{0,1\}$.

Received January 12, 1993; revised March 6, 1995 
An algebra $V=\left\langle V, \wedge^{*}, \vee^{*}\right\rangle$ is called a quasi-lattice iff for all $\alpha, \beta, \gamma \in V$ the following conditions are satisfied (see Płonka [43]):

1. $\alpha \wedge^{*} \alpha=\alpha \vee^{*} \alpha=\alpha$,

2. $\alpha \wedge^{*} \beta=\beta \wedge^{*} \alpha$, and $\alpha \vee^{*} \beta=\beta \vee^{*} \alpha$,

3. $\alpha \wedge^{*}\left(\beta \wedge^{*} \gamma\right)=\left(\alpha \wedge^{*} \beta\right) \wedge^{*} \gamma$ and $\alpha \vee^{*}\left(\beta \vee^{*} \gamma\right)=\left(\alpha \vee^{*} \beta\right) \vee^{*} \gamma$.

We say that a logic is well-quantified iff the corresponding algebra $\left\langle V, \wedge^{*}, \vee^{*}\right\rangle$ is a quasi-lattice. Note that the quantifiers $\forall$ and $\exists$ can be defined in a logic as generalized conjunction and disjunction iff the logic is well-quantified.

Since every lattice is a quasi-lattice, the class of truth-complete $\mathbf{C}$-extending well-quantified logics contains many well-known nonclassical logics whose conjunction and disjunction induce a lattice structure, e.g., all the finite-valued logics of Łukasiewicz [35], the functionally complete logics of Post [44], the class of logics $T_{\epsilon_{0,1}, 1}^{n+1}$ of Yablonskii [57, and the logics corresponding to the algebras of Moisil [36.

In our opinion, it is even more interesting to consider examples of truth-complete C-extending logics such that the algebra $\left\langle V, \wedge^{*}, \vee^{*}\right\rangle$ is a quasi-lattice but not a lattice: a well known example is Bochvar's three-valued logic $\mathbf{B}_{3}$ [9]. Other examples include the three-valued logic of Ebbinghaus [17], the logic D of Segerberg [53], which is a functional extension of Hallden's logic [31, and the finite-valued generalization of Bochvar's logic introduced by Grigolia and Finn [27]. Of related interest are also the three-valued logic of Hoogewijs 32], certain conditional logics (see Guzman [28] and Guzman and Squier [29]), and many of the significance logics considered by Goddard and Routley [24].

In Finn et al. [21], many-valued logics are classified in terms of the informal semantics arising from the algebraic properties of their logical operations: thus, a logic is said to be of the uncertainty type if the truth values different from true and false stand for degrees of uncertainty (fuzziness); alternatively, if these truth values stand for "defects of sense," then the resulting logics are called significance logics. Both classes are examples of logics of partially defined predicates. There are certain similarities between some notions from [21] and our definitions of truth-complete, $\mathbf{C}$ extending, and well-quantified logics.

Among the papers containing results related to ours, first of all we should mention Surma [56] and Rousseau [49]. In these papers the authors give algorithms for axiomatizing every finite-valued logic; using these axioms one can construct calculi of $n$-termed sequents and analytic tableaux in the style of Smullyan 54]. Hilbert-type calculi are not considered in [49] or [56].

The main result of our joint papers [2] and [5] is the specification of a general, effective method for constructing Hilbert-type first-order calculi for any truthcomplete $\mathbf{C}$-extending well-quantified logic. (Note that the condition of being wellquantified is not necessary for constructing propositional calculi.) Further, in the final sections of [2] and [5], we describe a general effective method to construct so-called "quasi-Hilbert"-type first-order calculi for arbitrary finite-valued logics. A general computational scheme for two-termed sequents in finite-valued logics is given in our papers [3] and [4]. In [4] one can find proofs of the analogues of some wellknown model-theoretic results for arbitrary finite-valued calculi. (Examples include the Łoś ultraproduct theorem, Maltsev's compactness theorem, and others.) In this 
connection, at least the following papers should also be mentioned: [2], [3], 44, [5], D'Ottaviano [40], [41], and [42], along with the abstracts [6] and [52].

Let us now mention the papers that were the sources of some of our ideas and methods. These papers are mainly concerned with logics where the algebra $\left\langle V, \wedge^{*}, \vee^{*}\right\rangle$ is a quasi-lattice but not a lattice (logics of significance, in the terminology of [21]). Bochvar's logic $\mathbf{B}_{3}$ from 91 is the first example. This logic and its corresponding class of algebras were axiomatized by Finn [19], [20], Bochvar and Finn [10]. Grigolia and Finn 22, 27] considered highly nontrivial logics and algebras yielding finite-valued generalizations of $\mathbf{B}_{3}$. The techniques developed by these two authors found subsequent applications in more general contexts. In particular, our formulations of logical and algebraic axioms are generalizations and simplifications of those in [22] and [27].

The main results of this paper are as follows: we introduce a general method of constructing Hilbert-type propositional calculi for all truth-complete $\mathbf{C}$-extending logics. We similarly construct "quasi-Hilbert"-type propositional calculi for all finite-valued logics. We prove the completeness of all these calculi. We study the relationship between classical validity and validity in truth-complete $\mathbf{C}$-extending logics. We describe the corresponding algebras of this class of logics and prove two representation theorems. Our proof of the completeness theorem is new: we construct realizations for formulas and valuations, following an idea of Skvortsov (see [7). The same method is used in the present paper to obtain various algebraic results.

Although all papers mentioned up to now are mainly concerned with finitevalued logics, let us also briefly mention some papers dealing with infinite-valued logics (and their corresponding algebras) having some relationship with the present paper. For example, in Rousseau [50], [51, and Girard [23], intuitionistic versions of finite-valued logics are considered. Such logics were also considered in Anshakov's dissertation [1. Unfortunately, some of Anshakov's papers about intuitionistic versions of finite-valued logics are quite inaccessible for western readers. Also the socalled J-defined logics from $[7]$ are closely related to the logics considered in the present paper. J-defined logics can be infinite-valued, as well as finite-valued. It is not hard to prove that every truth-complete $\mathbf{C}$-extending logic is J-defined.

Unless otherwise specified, by a logic $L_{n}$ we shall mean a truth-complete and C-extending logic given by a triple $\langle V, \sigma, \operatorname{MARK}\rangle$, where the signature

$$
\begin{aligned}
\sigma & =\sigma_{1} \cup\left\{\mathrm{J}_{\alpha}^{*} \mid \alpha \in V\right\} \cup\left\{\wedge^{*}, \vee^{*}, \supset^{*}, \neg^{*}\right\} \\
& =\left\langle\left\{\mathrm{J}_{\alpha}^{*} \mid \alpha \in V\right\}, \wedge^{*}, \vee^{*}, \supset^{*}, \neg^{*}, F_{1}, \ldots, F_{k}\right\rangle
\end{aligned}
$$

is functionally equivalent to the basic signature $\sigma_{1}$. In Section 4 we shall briefly discuss (along the lines of [2] and [5]) the possibility of extending our results to other classes of finite-valued logics. In Section 5 we shall describe $L_{n}$-derivable formulas of a truth-complete $\mathbf{C}$-extending finite-valued propositional calculus $L_{n}$ in an algebraic language.

Different algebraic approaches to various classes of many-valued logics are developed in several papers (notably Chang [13] and [14, Rasiowa [45], Cleave [16, Rosenberg [47], Cignoli [15], Grigolia [25, [26] and [27], Mundici [37, [38], and [39], Guzman and Squire [29, Komori [33] and [34], Rose and Rosser [46], 
Hähnle [30], Suchon [55], and Figallo [18]).

2 Syntax With the operations $\left\{\mathrm{J}_{\alpha}^{*} \mid \alpha \in V\right\}, \wedge^{*}, \vee^{*}, \supset^{*}, \neg^{*}$, and $F_{1}^{\left(m_{1}\right)}, \ldots, F_{k}^{\left(m_{k}\right)}$ we associate the formal symbols (called propositional connectives) $\left\{\mathrm{J}_{\alpha} \mid \alpha \in V\right\}, \wedge$, $\vee, \supset, \neg$ and $f_{1}^{\left(m_{1}\right)}, \ldots, f_{k}^{\left(m_{k}\right)}$, with the corresponding number of arguments.

Definition 2.1 (Alphabet and Formulas) We have:

1. A countable set of propositional variables,

2. Propositional connectives,

3. Parentheses ) and ( .

Formulas are defined in the usual way by induction. Propositional variables will be considered as atomic formulas.

We shall adopt the usual conventions on the omission of parentheses. All connectives in $\sigma \backslash\{\wedge, \vee, \supset\}$ have the same strength, and they are stronger than $\wedge, \vee, \supset$. The connectives $\wedge$ and $\vee$ are stronger than $\supset$.

Definition 2.2 External formulas are inductively defined as follows:

1. For each $\alpha \in V$, if $A$ is a formula, then $\mathrm{J}_{\alpha} A$ is an external formula.

2. If $X$ and $Y$ are external formulas, then so are $(X \wedge Y),(X \vee Y),(X \supset Y),(\neg X$.)

In what follows, the letters $p, q, r, \ldots$ shall stand for arbitrary atomic formulas, the letters $A, B, C, \ldots$ for formulas, and the letters $X, Y, Z, \ldots$ for external formulas.

Notation 2.3 Throughout this paper, $p_{0}$ shall denote a fixed propositional variable. We also use the following notations and abbreviations:

1. $\mathbf{T}=\left(\mathrm{J}_{1} p_{0} \supset \mathrm{J}_{1} p_{0}\right)$ and $\mathbf{F}=(\neg \mathbf{T})$,

2. $A \equiv B=(A \supset B) \wedge(B \supset A)$,

3. The abbreviations $\bigwedge_{i \in I} X_{i}$ and $\bigvee_{i \in I} X_{i}$ are interpreted, respectively, as $X_{1} \wedge\left(X_{2} \wedge\right.$ $\left.\left.\ldots \wedge\left(X_{k-1} \wedge X_{k}\right) \ldots\right)\right)$ and $\left.X_{1} \vee\left(X_{2} \vee \ldots \vee\left(X_{k-1} \vee X_{k}\right) \ldots\right)\right)$, under the assumption that $I=\{1, \ldots, k\}$. If $I=\varnothing$, then $\mathbf{T}$ is identified with the formula $\bigwedge X_{i}$, and $\mathbf{F}$ with the formula $\bigvee_{i \in I} X_{i}$. As for the binary operations $\wedge^{*}$ and $\vee^{*}$, the expressions $\bigwedge_{\beta \in I}^{*} \beta$ and $\bigvee_{\beta \in I}^{*} \beta$ are handled in the same way, with $\bigwedge_{\beta \in I}^{*} \beta=1$ and $\bigvee_{\beta \in I}^{*} \beta=0$ for $I=\varnothing$.

4. If $W \subseteq V$, then we set $\mathrm{J}_{W} A=\bigvee_{\beta \in W} \mathrm{~J}_{\beta} A$. Let mark $A=\mathrm{J}_{\mathrm{MARK}} A$.

Definition 2.4 We adopt the following Axiom Schemes:

Propositional axioms (P)

(P1) $\quad X \supset(Y \supset X)$,

(P2) $\quad(X \supset(Y \supset Z)) \supset((X \supset Y) \supset(X \supset Z))$,

(P3a) $X \supset(Y \supset X \wedge Y)$,

(P3b) $X \wedge Y \supset X$,

(P3c) $X \wedge Y \supset Y$, 
(P4a) $(X \supset Z) \supset((Y \supset Z) \supset(X \vee Y \supset Z))$,

(P4b) $X \supset X \vee Y$,

(P4c) $\quad Y \supset X \vee Y$,

(P5) $(X \supset Y) \supset((X \supset \neg Y) \supset \neg X)$,

(P6) $\neg \neg X \supset X$.

\section{Connection Axioms (Con)}

(Cg) $\quad \mathrm{J}_{\alpha} g\left(A_{1}, \ldots, A_{r}\right) \equiv \underset{G\left(\beta_{1}, \ldots, \beta_{r}\right)=\alpha}{\bigvee}\left(\bigwedge_{i=1}^{r} \mathrm{~J}_{\beta_{i}} A_{i}\right)$,

where $G \in \sigma \backslash\left\{J_{\alpha}^{*} \mid \alpha \in V\right\}$ and the propositional connective $g$ corresponds to the operation $G$,

$\left(\mathbf{C J}_{0}\right) \quad \mathrm{J}_{0} X \equiv \neg X$,

$\left(\mathbf{C J}_{1}\right) \quad \mathrm{J}_{1} X \equiv X$

$\left(\mathbf{C J}_{\alpha}\right) \quad \mathbf{J}_{\alpha} X \supset \mathbf{F}$ for $\alpha \notin\{0,1\}$.

\section{Axioms of $n$-valuedness}

For each $\alpha \in V$ :

$\left(\mathrm{N} V_{\alpha}\right) \mathrm{J}_{\alpha} A \equiv \bigwedge_{\beta \neq \alpha} \neg \mathrm{J}_{\beta} A$.

Definition 2.5 We use the following inference rules:

1. modus ponens: $\frac{Y, \quad X \supset Y}{Y}$

2. mark-introduction: $\frac{A}{\operatorname{mark} A}$

3. mark-elimination: $\frac{\operatorname{mark} A}{A}$

As usual, a formula $A$ is said to be derivable from a collection of formulas $\Gamma$ in the calculus $L_{n}$ if it is derivable from the axioms and $\Gamma$ by the rules of inference (notation: $\Gamma \vdash{ }_{L_{n}} A$, or simply $\Gamma \vdash A$.)

Let $\mathbf{E F}\left(L_{n}\right)$ be the set of all external formulas of the calculus $L_{n}$. Let $\mathbf{P V}(\mathbf{C})$ be the set of all propositional variables of the classical propositional calculus $\mathbf{C}$, and let $\mathbf{A F}(\mathbf{C})$ be the algebra of formulas of the logic $\mathbf{C}$, that is, the absolutely free algebra of signature $\{\wedge, \vee, \supset, \neg\}$ generated by $\mathbf{P V}(\mathbf{C})$. We also denote by $\mathbf{A F}(\mathbf{C})$ the support of this algebra.

Proposition 2.6 Let $S:\langle\mathbf{A F}(\mathbf{C}), \wedge, \vee, \supset, \neg\rangle \longrightarrow\left\langle\mathbf{E F}\left(L_{n}\right), \wedge, \vee, \supset, \neg\right\rangle$ be a homomorphism. By abuse of notation, let $S: \mathbf{P V}(\mathbf{C}) \longrightarrow \mathbf{E F}\left(L_{n}\right)$ also denote the restriction of $S$ to $\mathbf{P V}(\mathbf{C})$. If $\Phi \in \mathbf{A F}(\mathbf{C})$ and $\Gamma \subseteq \mathbf{A F}(\mathbf{C})$, then $\Gamma \vdash_{\mathbf{C}} \Phi$ implies $S(\Gamma) \vdash{ }_{L_{n}} S(\Phi)$, where $S(\Gamma)=\{S(\Psi) \mid \Psi \in \Gamma\}$.

Proof: An obvious induction on the length of the derivation of the formula $\Phi$ in the classical propositional calculus $\mathbf{C}$. 
It follows from the above proposition that we can use well-known derived rules of inference of the classical logic $\mathbf{C}$ for constructing derivations in the calculus $L_{n}$, provided that we restrict the area of their application to the $\operatorname{set} \mathbf{E F}\left(L_{n}\right)$ of external formulas.

Lemma 2.7 If $1 \in W \subseteq V$ and $0 \notin W$, then $\vdash_{L_{n}} J_{W} X \supset X$ and $\vdash_{L_{n}} X \supset J_{W} X$.

Proof: (a) Let us prove that $\vdash X \supset \mathbf{J}_{W} X$. By axiom $\left(\mathbf{C J}_{1}\right)$ and Proposition 2.6, we have $\vdash X \supset \mathrm{J}_{1} X$. Moreover, $\vdash \mathrm{J}_{1} X \supset \mathrm{J}_{W} X$ because $1 \in W$. Then we have $\vdash X \supset$ $\mathrm{J}_{W} X$.

(b) Let us prove $\vdash \mathrm{J}_{W} X \supset X$. By axiom $\left(\mathbf{C J}_{1}\right)$ and Proposition 2.6. we have $\vdash \mathrm{J}_{1} X \supset X$. If $1 \neq \alpha \in W$ then by axiom $\left(\mathbf{C J}_{\alpha}\right)$ we have $\vdash \mathbf{J}_{\alpha} X \supset \mathbf{F}$ because $\alpha \neq 0$. Again by Proposition 2.6 we get $\vdash\left(\mathrm{J}_{\alpha} X \supset \mathbf{F}\right) \supset\left(\mathrm{J}_{\alpha} X \supset X\right)$. Then, by modus ponens, $\vdash \mathrm{J}_{\alpha} X \supset X$ for $1 \neq \alpha \in W$. So for all $\alpha \in W$ we have $\vdash \mathrm{J}_{\alpha} X \supset X$, which implies $\vdash \mathrm{J}_{W} X \supset X$.

Corollary $2.8 \vdash_{L_{n}} X \equiv \operatorname{mark} X$.

Theorem 2.9 (Deduction Theorem) Let $\Gamma$ be a collection offormulas, and suppose $A$ and $B$ to be formulas of $L_{n}$. Then $\Gamma, A \vdash_{L_{n}} B$ implies $\Gamma \vdash_{L_{n}}$ mark $A \supset$ mark $B$.

Proof: By induction on the length of the derivation of the formula $B$, as in classical logic. If necessary, use Proposition 2.6 and the above corollary.

Corollary 2.10 If $X$ and $Y$ are external formulas, then $\Gamma, X \vdash_{L_{n}} Y$ implies $\Gamma \vdash_{L_{n}}$ $X \supset Y$.

Definition 2.11 We define normal formulas by the following inductive procedure:

1. For $\alpha \in V$, if $A$ is an atomic formula, then $\mathrm{J}_{\alpha} A$ is a normal formula.

2. If $X$ and $Y$ are normal formulas, then so are the expressions $(X \wedge Y),(X \vee$ $Y),(X \supset Y)$, and $(\neg X)$.

Definition 2.12 By induction we define the I operator, transforming external formulas into normal formulas, as follows.

1. If $A$ is an atomic formula, then $\left(\mathrm{J}_{\alpha} A\right)^{I}=\mathrm{J}_{\alpha} A$.

2. $(X * Y)^{I}=X^{I} * Y^{I}$, where $*$ here denotes one of the connectives, $\vee, \wedge, \supset$.

3. $(\neg X)^{I}=\neg\left(X^{I}\right)$.

4. $\left(\mathbf{J}_{\alpha} f\left(A_{1}, \ldots, A_{r}\right)\right)^{I}=\underset{F\left(\beta_{1}, \ldots, \beta_{r}\right)=\alpha}{\bigvee}\left(\bigwedge_{j=1}^{r}\left(\mathrm{~J}_{\beta_{j}} A_{j}\right)^{I}\right)$, where the connective $f$ 5. corresponds to the operation $F \in \sigma \backslash\left\{\mathrm{J}_{\alpha}^{*} \mid \alpha \in V\right\}$,

$$
\left(\mathrm{J}_{\alpha} \mathrm{J}_{\beta} A\right)^{I}= \begin{cases}\left(\mathrm{J}_{\beta} A\right)^{I} & \text { if } \alpha=1, \\ \neg\left(\mathrm{J}_{\beta} A\right)^{I} & \text { if } \alpha=0, \\ \mathbf{F} & \text { if } \alpha \notin\{0,1\}\end{cases}
$$

Lemma 2.13 $\vdash_{L_{n}} X \equiv X^{I}$. 
Proof: By induction on the logical depth of the external formula $X$. Use Proposition 2.6 and the axioms of connection (Con).

Corollary 2.14 $\vdash_{L_{n}} A$ if and only if $\vdash_{L_{n}}(\text { mark } A)^{I}$.

3 Semantics Recall that $\mathbf{A F}\left(L_{n}\right)$ denotes the algebra of formulas of our logic $L_{n}$ (that is, the absolutely free algebra of signature $\sigma$ ). Homomorphisms from the algebra of formulas $\mathbf{A F}\left(L_{n}\right)$ into the algebra $\langle V, \sigma\rangle$ will be called $L_{n}$-valuations.

Lemma 3.1 Let $X$ be an external formula of the calculus $L_{n}$. If $v$ is an $L_{n^{-}}$ valuation, then $v(X) \in\{0,1\}$.

Proof: By an obvious induction on the construction of the formula $X$.

Definition 3.2 A formula $A$ of the language $L_{n}$ is said to be $L_{n}$-valid if we have $v(A) \in$ MARK for each $L_{n}$-valuation $v$.

The following is an immediate consequence of the definition.

Lemma 3.3 Let $v$ be an arbitrary $L_{n}$-valuation and $W \subseteq V$. Then

$$
v\left(J_{W} A\right)= \begin{cases}1 & \text { if } v(A) \in W \\ 0 & \text { if } v(A) \notin W .\end{cases}
$$

Corollary 3.4 A formula A of the language $L_{n}$ is $L_{n}$-valid if and only if the formula mark $A$ is $L_{n}$-valid.

Theorem 3.5 (Correctness Theorem) All formulas derivable in the calculus $L_{n}$ are $L_{n}$-valid.

Proof: It suffices to verify that the axioms are $L_{n}$-valid and the rules of inference preserve $L_{n}$-validity. The fact that modus ponens preserves $L_{n}$-validity is clear. For the rules of mark-introduction and mark-elimination, this follows from Corollary 3.4.

The $L_{n}$-validity of axioms of group (P) follows from Lemma 3.1. since the restrictions of the operations $\wedge^{*}, \vee^{*}, \supset^{*}, \neg^{*}$ to the subset $\{0,1\}$ of the set $V$ are the usual operations of conjunction, disjunction, implication, and negation, respectively. The $L_{n}$-validity of the other axioms can be directly verified.

Lemma 3.6 A formula $A$ is $L_{n}$-valid if and only if the normal formula (mark $\left.A\right)^{I}$ is $L_{n}$-valid.

Proof: Use Corollary 3.4. together with the Correctness Theorem 3.5 and Lemma 2.13 .

Let $\mathbf{P V}\left(L_{n}\right)$ denote the set of all propositional variables of the logic $L_{n}$. We shall present the set $\mathbf{P V}\left(L_{n}\right)$ as a disjoint union of $n$-element subsets of $\mathbf{P V}(\mathbf{C})$. To this purpose, we assume to be given, once and for all, a one-to-one correspondence between the set $\mathbf{P V}\left(L_{n}\right)$ and the set of all $n$-element subsets of $\mathbf{P V}(\mathbf{C})$. So each propositional variable $p \in \mathbf{P V}\left(L_{n}\right)$ will correspond to some fixed collection of $n$ propositional variables from $\mathbf{P V}(\mathbf{C})$. For convenience, we shall denote the elements in this collection 
as propositional letters indexed by the elements of the set $V$ of truth values. So we have

$$
\mathbf{P V}(\mathbf{C})=\bigcup_{p \in \mathbf{P V}\left(L_{n}\right)}\left\{p_{\alpha} \mid \alpha \in V\right\}
$$

where $\left\{p_{\alpha} \mid \alpha \in V\right\} \cap\left\{q_{\alpha} \mid \alpha \in V\right\}=\varnothing$ for $p \neq q$, and $p_{\alpha} \neq p_{\beta}$ whenever $\alpha \neq \beta$.

We denote by $\mathbf{P V}(A)$ the set of all propositional variables of a formula $A$.

Definition 3.7 The expression $X^{R}$ denotes the formula of classical logic obtained from a normal formula $X$ by substituting $p_{\alpha}$ for all occurrences of $\mathrm{J}_{\alpha} p$. We shall call this classical formula $X^{R}$ the realization of the formula $X$.

Definition 3.8 Let $v$ be an $L_{n}$-valuation. Let us define a classical valuation $v_{R}$ as follows: we set $v_{R}\left(p_{\alpha}\right)=v\left(\mathrm{~J}_{\alpha} p\right)$, and then we extend this valuation to the set $\mathbf{A F}(\mathbf{C})$ of all formulas of the classical logic $\mathbf{C}$. We shall call this valuation $v_{R}$ the realization of the $L_{n}$-valuation $v$.

Lemma 3.9 Let $v$ be an $L_{n}$-valuation and $X$ be a normal formula. Then $v(X)=$ $v_{R}\left(X^{R}\right)$.

Proof: By an obvious induction on the construction of the formula $X$.

We now define

$$
(N V)_{A}=\bigwedge_{\alpha \in V}\left(\mathrm{~J}_{\alpha} A \equiv \bigwedge_{\beta \neq \alpha} \neg \mathrm{J}_{\beta} A\right) .
$$

Compare with the axioms $\left(N V_{\alpha}\right)$.

Definition 3.10 A classical valuation $u$ will be called normal if for every $p \in$ $\mathbf{P V}\left(L_{n}\right)$ we have $u\left((N V)_{p}^{R}\right)=1$.

Lemma 3.11 If $v$ is an $L_{n}$-valuation, then $v_{R}$ is a normal $\mathbf{C}$-valuation.

Proof: By Lemma 3.9 together with the Correctness Theorem 3.5, we have $v_{R}\left((N V)_{p}^{R}\right)=v\left((N V)_{p}\right)=1$.

Lemma 3.12 For every normal $\mathbf{C}$-valuation $u$ there exists an $L_{n}$-valuation $v$ such that $u=v_{R}$.

Proof: $\quad$ Since $u\left((N V)_{p}^{R}\right)=1$, there exists a unique $\alpha \in V$ such that $u\left(p_{\alpha}\right)=1$. Letting now $v(p)=\alpha$, we extend (by induction) this map to an $L_{n}$-valuation. Then it is easy to see that $v_{R}=u$.

Lemma 3.13 A normal formula $X$ is $L_{n}$-valid if and only if for every normal $\mathbf{C}$ valuation $u$ it is true that $u\left(X^{R}\right)=1$.

Proof: $\quad$ Let $X$ be $L_{n}$-valid and $u$ be a normal C-valuation. By Lemma 3.12 there exists an $L_{n}$-valuation $v$ such that $u=v_{R}$. By Lemma 3.9. we have $u\left(X^{R}\right)=v_{R}\left(X^{R}\right)=$ $v(X)=1$. Assume $u\left(X^{R}\right)=1$ holds for every normal $\mathbf{C}$-valuation $u$. Then by Lemma 3.11. for every $L_{n}$-valuation $v, v_{R}$ is a normal C-valuation. By Lemma 3.9. we conclude $v(X)=v_{R}\left(X^{R}\right)=1$.

In the rest of this paper we let $p_{0} \in \mathbf{P V}\left(L_{n}\right)$ be the same distinguished propositional variable used in our notational stipulations 2.3 . 
Lemma 3.14 Let $X$ be a normal formula. Let $\wp$ be a finite subset of the set $\mathbf{P V}\left(L_{n}\right)$ such that $\mathbf{P V}(X) \subseteq \wp$ and $p_{0} \in \wp$. Then $X$ is $L_{n}$-valid if and only if the formula

$$
\bigwedge_{p \in \wp}(N V)_{p}^{R} \supset X^{R}
$$

is a classical tautology.

Proof: $\quad$ Let a normal formula $X$ be $L_{n}$-valid. Let $u$ be an arbitrary $\mathbf{C}$-valuation. Assume we have

$$
u\left(\bigwedge_{p \in \wp}(N V)_{p}^{R}\right)=1 .
$$

Let us define a $\mathbf{C}$-valuation $w$ as follows. If $p \in \wp$, then we set $w\left(p_{\alpha}\right)=u\left(p_{\alpha}\right)$; in the opposite case we set

$$
w\left(p_{\alpha}\right)= \begin{cases}1 & \text { if } \alpha=1 \\ 0 & \text { if } \alpha \neq 1\end{cases}
$$

It is easy to see that $w$ is a normal $\mathbf{C}$-valuation. Moreover, if all propositional variables occurring in a classical formula $\Phi$ are contained in the set $\left\{p_{\alpha} \mid p \in \wp, \alpha \in V\right\}$, then $w(A)=u(A)$. Then, by Lemma 3.13. we have $u\left(X^{R}\right)=w\left(X^{R}\right)=1$.

Let the formula (1) be a classical tautology and $v$ be an arbitrary $L_{n}$-valuation. Let us set $u=v_{R}$. Then, by Lemma 3.11. $u$ is a normal $\mathbf{C}$-valuation. Therefore, $u\left(X^{R}\right)=1$. Then $v(X)=u\left(X^{R}\right)=1$.

Corollary 3.15 (Embedding of $L_{n}$ into classical logic $\mathbf{C}$ ) Let $A$ be a formula of the logic $L_{n}, \mathbf{P V}(A) \subseteq \wp \subseteq \mathbf{P V}\left(L_{n}\right)$, where $\wp$ is a finite set, and $p_{0} \in \wp$. Then $A$ is $L_{n}$ valid if and only if the formula

$$
\bigwedge_{p \in \wp}(N V)_{p}^{R} \supset\left((\operatorname{mark} A)^{I}\right)^{R}
$$

is a classical tautology.

Proof: Use Lemmas 3.6 and 3.13.

Theorem 3.16 (Completeness Theorem) If a formula A of the language $L_{n}$ is $L_{n}$ valid, then $A$ is derivable in the calculus $L_{n}$.

Proof: $\quad$ Let $\wp=\mathbf{P V}(A) \cup\left\{p_{0}\right\}$. Let $A$ be $L_{n}$-valid. By Corollary 3.15 and the Completeness Theorem for the classical logic $\mathbf{C}$, we have

$$
\vdash_{\mathbf{C}} \bigwedge_{p \in \wp}(N V)_{p}^{R} \supset\left((\operatorname{mark} A)^{I}\right)^{R} .
$$

Then, by Proposition 2.6, we have

$$
\vdash_{L_{n}} \bigwedge_{p \in \wp}(N V)_{p} \supset(\operatorname{mark} A)^{I} .
$$

If $p \in \wp$, then $(N V)_{p}$ is the axiom of the calculus $L_{n}$. By modus ponens, we have $\vdash_{L_{n}}(\text { mark } A)^{I}$. Then, by Lemma 2.13 and the mark-elimination rule, we have $\vdash_{L_{n}}$ A. 
Definition 3.17 For convenience, now we shall consider that $\mathbf{P V}(\mathbf{C})=\left\{q_{m} \mid m \in \omega\right\}$ and $\mathbf{P V}\left(L_{n}\right)=\left\{p_{m} \mid m \in \omega\right\}$. Let $u$ be a $\mathbf{C}$-valuation. We set $u_{n}\left(p_{m}\right)=u\left(q_{m}\right)$, and then we shall extend $u_{n}$ to $L_{n}$-valuation by the usual inductive procedure. This $L_{n}$ valuation $u_{n}$ will be called the $L_{n}$-version of the $\mathbf{C}$-valuation $u$.

Definition 3.18 The expression $\Phi^{B}$ denotes the external formula of the logic $L_{n}$ obtained from a formula $\Phi$ of classical logic $\mathbf{C}$ by substituting $\mathrm{J}_{1} D$ for all occurrences of every atomic subformula $D$ of the formula $\Phi$. This formula $\Phi^{B}$ is called the Bochvar version of the formula $\Phi$.

Lemma 3.19 Let $v$ be a $\mathbf{C}$-valuation and $\Phi$ be a formula of the classical logic $\mathbf{C}$. Then $v(\Phi)=v_{n}\left(\Phi^{B}\right)$.

Proof: By an obvious induction on the complexity of $\Phi$, recalling Lemma 3.1

Theorem 3.20 (Embedding of classical logic $\mathbf{C}$ into $L_{n}$ ) Let $\Phi$ be a formula of classical logic C. Then $\vdash_{\mathbf{C}} \Phi$ if and only if $\vdash_{L_{n}} \Phi^{B}$.

Proof: By Proposition 2.6. $\vdash$ C $\Phi$ implies $\vdash_{L_{n}} \Phi^{B}$. If $\vdash_{L_{n}} \Phi^{B}$, then $\Phi^{B}$ is $L_{n}$-valid by the Correctness Theorem for the calculus $L_{n}$. Let $v$ be an arbitrary $\mathbf{C}$-valuation. Then $v(\Phi)=v_{n}\left(\Phi^{B}\right)=1$. Therefore, $\vdash_{\mathbf{C}} \Phi$ follows from the Completeness Theorem for classical logic $\mathbf{C}$.

4 Axiomatizing arbitrary finite-valued logics We note that, for an arbitrary finitevalued logic $L_{n}$ given by a triple $\langle V, \sigma, \mathrm{MARK}\rangle$ there is no known effective method for constructing a calculus either in the Hilbert form or in the form of a calculus of two-termed sequents, which will be complete with respect to $L_{n}$-validity. Admittedly, Rousseau [49] and Surma [56] developed methods of axiomatizing finite-valued logical calculi, but although these methods may be of theoretical value, it seems to us that they are too complex and unintuitive.

Investigators of finite-valued logical calculi usually strive to obtain complete (with respect to $L_{n}$-validity) axiomatizations of predicate or propositional manyvalued calculi, either of the Hilbert type, or in the form of two-termed sequents. In this way one has the opportunity to obtain deeper proof-, as well as model-theoretic results: as a matter of fact, these methods of axiomatization are nicely linked with natural mathematical intuition, and they also enable us to extend to finite-valued logical calculi methods and results that were originally developed for classical logic. This is the case, e.g., of the axiomatizations for logics of Łukasiewicz in 35, Bochvar [9], 24], Ebbinghaus [17], Rose and Rosser [46], and others.

In our papers [2], [3], 4], [5] we presented a general effective method for constructing a predicate calculus of "quasi-Hilbert" type, which is complete with respect to $L_{n}$-validity, for any finite-valued logic. It appears that in many problems arising for finite-valued calculi, "quasi-Hilbert"-type calculi are no less convenient than Hilberttype calculi-the only difference being the addition of finitely many symbols in the language. Once the language is so extended, all subsequent reasoning is carried out in a Hilbert type calculus. We shall briefly discuss this idea for propositional calculi only. 
Let the logic $L_{n}$ be given by a triple $\left\langle V, \sigma_{1}\right.$, MARK $\rangle$. We extend the alphabet of $L_{n}$ by introducing the following new symbols: the external connectives, $\wedge, \vee, \supset, \neg$, and $\left\{J_{\alpha} \mid \alpha \in V\right\}$. Formulas and external formulas are defined as in Section 2. Thus, although formulas will be in the language of the logic $L_{n}$, external formulas will not be in it. For the axiomatization of this extended calculus we take all the axioms from Section 2 (excluding the axioms $\left(\mathbf{C J}_{0}\right),\left(\mathbf{C J}_{1}\right)$ and $\left(\mathbf{C J}_{\alpha}\right)$ ). Moreover, we shall assume that the axioms $(\mathbf{C g})$ are valid for all $G \in \sigma_{1} \cup\left\{\wedge^{*}, \vee^{*}, \supset^{*}, \neg^{*}\right\}$, where $\wedge^{*}, \vee^{*}, \supset^{*}, \neg^{*}$ are usual two-valued logical operations on the subset $\{0,1\}$ of the set $V$ of truth values; further, we shall maintain all conventions of Section 2 about metasymbols for formulas and external formulas. We denote this extended calculus by $L_{n}(+)$. It is convenient to regard our calculus $L_{n}(+)$ as the counterpart of the twosorted algebra $\left\langle V,\{0,1\}, \sigma_{1}, \wedge^{*}, \vee^{*}, \supset^{*}, \neg^{*},\left\{J_{\alpha}^{*} \mid \alpha \in V\right\}\right\rangle$. It is possible to define $L_{n}(+)$-valuations of formulas and external formulas in the usual way. We set

$$
\Phi^{M}= \begin{cases}(\text { mark } \Phi) & \text { if } \Phi \text { is a formula, } \\ \Phi & \text { if } \Phi \text { is an external formula }\end{cases}
$$

It is not difficult to check that all results from Sections 2 and 3 (except Lemma 2.7 and Corollary 2.8 are true for the calculus $L_{n}(+)$. To see this, one may proceed as follows:

1. If necessary, substitute $A^{M}$ for mark $A$ (see for example, in 2.9.2.14. 3.4. 3.6. and 3.15),

2. Exclude all cases dealing with formulas $\left(\mathrm{J}_{\alpha} X\right)$, where $X$ is an external formula, since now the expressions of the type $\left(\mathrm{J}_{\alpha} X\right)$ are not formulas or external formulas of our calculus $L_{n}(+)$. Of course, the relevant definitions must be correspondingly adapted: for example, the fifth item in Definition 2.12 must be eliminated.

Repeating (with natural modifications) the arguments of Sections 2 and 3, one can see that the following result holds.

Theorem 4.1 (Completeness Theorem) Let $\Phi$ be a formula or an external formula of the calculus $L_{n}(+)$. Then $\vdash_{L_{n}(+)} \Phi$ if and only if $\Phi$ is $L_{n}(+)$-valid.

Remark 4.2 A formula $A$ of the calculus $L_{n}(+)$ is $L_{n}(+)$-valid if and only if $A$ is $L_{n}$-valid, since $A$ is written in the language $L_{n}$.

A modified method of sequents and tableaux systems with additional logical metasymbols was recently used in Carnielli [11] and [12] for the axiomatization of calculi for various finite-valued logics.

5 An algebraic approach to finite-valued logics Let $L_{n}$ be an arbitrary truthcomplete $\mathbf{C}$-extending logic determined by the triple $\langle V, \sigma$, MARK $\rangle$.

\section{Definition 5.1}

1. For an arbitrary Boolean algebra $\langle B, \cap, \cup,-, 0,1\rangle$ we shall use the following standard abbreviations:

(a) $a \rightarrow b=-a \cup b$, 
(b) $a \leftrightarrow b=(a \rightarrow b) \cap(b \rightarrow a)$.

2. We define the sets

$$
\hat{B}=\left\{x \in B^{V} \mid x(\alpha)=\bigcap_{\beta \neq \alpha}-x(\beta) \text { for each } \alpha \in V\right\},
$$

where $x(\alpha)$ denotes the $\alpha$ th coordinate of $x \in B^{V}$, and

$$
\operatorname{MARK}(\hat{B})=\left\{x \in \hat{B} \mid \bigcup_{\alpha \in \operatorname{MARK}} x(\alpha)=1\right\} .
$$

3. For each $m$-ary operation $G \in \sigma$ we define the operation $\hat{G}$ on $B^{V}$ as follows:

(a) For $x_{1}, \ldots, x_{m} \in B^{V}$ and $G \in \sigma \backslash\left\{\mathrm{J}_{\beta}^{*} \mid \beta \in V\right\}$ we set

$$
\left(\hat{G}\left(x_{1}, \ldots, x_{m}\right)\right)(\alpha)=\bigcup_{G\left(\beta_{1}, \ldots, \beta_{m}\right)=\alpha}\left(\bigcap_{j=1}^{m} x_{j}\left(\beta_{j}\right)\right),
$$

(b) For $x \in B^{V}$ we set

$$
\left(\hat{\mathbf{J}}_{\beta} x\right)(\alpha)=\left\{\begin{aligned}
x(\beta) & \text { if } \alpha=1, \\
-x(\beta) & \text { if } \alpha=0, \\
0 & \text { if } \alpha \notin\{0,1\} .
\end{aligned}\right.
$$

4. For each $a \in B$, we define the element $\hat{a} \in B^{V}$ as follows:

$$
\hat{a}(\alpha)=\left\{\begin{aligned}
a & \text { if } \alpha=1 \\
-a & \text { if } \alpha=0, \\
0 & \text { if } \alpha \notin\{0,1\} .
\end{aligned}\right.
$$

5. For $x \in B^{V}$ we shall use the following abbreviation:

$$
[[N V]]_{x}=\bigcap_{\alpha \in V}\left(x(\alpha) \leftrightarrow \bigcap_{\beta \neq \alpha}-x(\beta)\right) .
$$

Proposition 5.2 Let $\langle B, \cap, \cup,-, 0,1\rangle$ be a Boolean algebra. Then for any $x \in B^{V}$ the following three conditions are equivalent:

1. $x \in \hat{B}$,

2. $\bigcup_{\alpha \in V} x(\alpha)=1$, and $x(\alpha) \cap x(\beta)=0$ for $\alpha \neq \beta$.

3. $[[N V]]_{x}=1$.

Proof: The fact that (1) is equivalent to (3) follows from the observation that $a \rightarrow$ $b=1$ if and only if $a \leq b$. Let us prove that (1) and (2) are equivalent. Let (1) be true. Then

$$
\begin{aligned}
\bigcup_{\alpha \in V} x(\alpha) & =x(\alpha) \cup\left(\bigcup_{\alpha \neq \beta} x(\beta)\right) \\
& =\left(-\bigcup_{\beta \neq \alpha} x(\beta)\right) \cup\left(\bigcup_{\beta \neq \alpha} x(\beta)\right)=1 .
\end{aligned}
$$


Moreover, if $\alpha \neq \beta$, then

$$
x(\alpha) \cap x(\beta)=\left(\bigcap_{\beta \neq \alpha}-x(\beta)\right) \cap x(\beta)=0 .
$$

Now let (2) be true. If $\alpha \neq \beta$, then $x(\alpha) \cap x(\beta)=0$ implies $x(\alpha) \leq-x(\beta)$. Therefore,

$$
x(\alpha) \leq \bigcap_{\beta \neq \alpha}-x(\beta) .
$$

On the other hand,

$$
\bigcup_{\alpha \in V} x(\alpha)=1
$$

implies

$$
\bigcap_{\beta \neq \alpha}-x(\beta) \leq x(\alpha)
$$

In conclusion, $x \in \hat{B}$.

Corollary 5.3 If $a \in B$, then $\hat{a} \in \hat{B}$.

Remark 5.4 $\hat{1} \in \operatorname{MARK}(\hat{B})$ and $\hat{0} \notin \operatorname{MARK}(\hat{B})$.

Proposition 5.5 The set $\hat{B}$ is closed under the operations $\{\hat{G} \mid G \in \sigma\}$.

Proof: $\quad$ Let $G \in \sigma \backslash\left\{\mathrm{J}_{\alpha}^{*} \mid \alpha \in V\right\}$ be an arbitrary $m$-ary operation and $x_{1}, \ldots, x_{m} \in \hat{B}$. Let $A$ be a formula $g\left(p_{1}, \ldots, p_{m}\right)$ of the calculus $L_{n}$, where the propositional connective $g$ corresponds to the operation $G$, and $p_{1}, \ldots, p_{m}$ are different propositional variables of $L_{n}$ (each of them not coinciding with $p_{0}$ ). Let $X$ be a formula $\left((N V)_{A}\right)^{I}$ (see Definition 2.12). The normal formula $X$ is $L_{n}$-derivable, by the Axioms of Definition 2.4. And therefore, by Theorem 3.5. Lemma 3.14 and the Completeness Theorem for classical logic, the formula

$$
\bigwedge_{j=0}^{m}(N V)_{p}^{R} \supset X^{R}
$$

is derivable in the classical propositional calculus. Therefore, for every homomorphism $h: \mathbf{A F}(\mathbf{C}) \rightarrow\langle B, \cap, \cup,-, \rightarrow\rangle$ the image of this formula is equal to 1 . Recalling our stipulation made above, we shall consider a homomorphism $h$ such that $h\left(p_{j_{\alpha}}\right)=x_{j}(\alpha)$ for $j=1, \ldots, m$. Let $x_{0}=\hat{1}$. Then by Corollary 5.3 and Proposition 5.2. we have

$$
h\left(\bigwedge_{j=0}^{m}(N V)_{P_{j}}^{R}\right)=\bigcap_{j=0}^{m}\left([[N V]]_{x_{j}}\right)=1 .
$$

Therefore, $[[N V]]_{\hat{G}\left(x_{1} \ldots, x_{m}\right)}=h\left(X^{R}\right)=1$. By Proposition 5.2. this means that $\hat{G}\left(x_{1}, \ldots, x_{m}\right) \in \hat{B}$. The set $\hat{B}$ is closed under the operations $\left\{\hat{\mathrm{J}}_{\beta} \mid \beta \in V\right\}$, by Corollary 5.3. since $\hat{\mathbf{J}}_{\beta} x=x \hat{(\beta)}$ for $x \in \hat{B}$. 
Definition 5.6 We call the algebra $\langle\hat{B},\{\hat{G} \mid G \in \sigma\}\rangle$ the $L_{n}$-version of the Boolean algebra $\langle B, \cap, \cup,-, 0,1\rangle$, and denote it by $B\left(L_{n}\right)$.

\section{Lemma 5.7}

1. The $L_{n}$-version $B\left(L_{n}\right)$ of every Boolean algebra $B$ contains an isomorphic copy of the algebra $\langle V, \sigma\rangle$ of the logic $L_{n}$;

2. If the Boolean algebra $B_{2}$ consists of the two elements 0 and 1 , then there exists an isomorphism from the algebra $\langle V, \sigma\rangle$ onto the algebra $B_{2}\left(L_{n}\right)$ such that the set MARK $\left(\hat{B}_{2}\right)$ is the image of the set MARK.

Proof: We define a (+)-map from $V$ to $B^{V}$ as follows: if $\alpha \in V$, then we set

$$
\alpha^{+}(\beta)= \begin{cases}1 & \text { if } \beta=\alpha \\ 0 & \text { if } \beta \neq \alpha\end{cases}
$$

By Proposition 5.2.2, we have $\left\{\beta^{+} \mid \beta \in V\right\} \subseteq \hat{B}$. Letting $x \in \hat{B}$ and $\operatorname{Rang}(x) \subseteq$ $\{0,1\}$, we also have

$$
\bigcup_{\alpha \in V} x(\alpha)=1
$$

whence there exists an $\alpha_{0}$ such that $x\left(\alpha_{0}\right)=1$. If $\beta \neq \alpha_{0}$, then (again by Proposition 5.2.2) we have $x(\beta)=0$. So, $x=\alpha_{0}^{+}$. This shows that $\left\{\beta^{+} \mid \beta \in V\right\}=$ $\{x \in \hat{B} \mid \operatorname{Rang}(x) \subseteq\{0,1\}\}$.

To see that the set $\left\{\beta^{+} \mid \beta \in V\right\}$ is closed under the operations $\{\hat{G} \mid G \in \sigma\}$, it suffices to prove that the results of these operations on the elements $\left\{\beta^{+} \mid \beta \in V\right\}$ are $\{0,1\}$-valued functions. But this follows from the definitions of the operations $\{\hat{G} \mid G \in \sigma\}$, and the fact that the set $\{0,1\}$ is the support of a two-element subalgebra of the Boolean algebra $B$. Thus, the set $\left\{\beta^{+} \mid \beta \in V\right\}$ is the support of some subalgebra of the algebra $B\left(L_{n}\right)$.

It is clear that the (+)-map is a one-to-one correspondence between the sets $V$ and $\left\{\beta^{+} \mid \beta \in V\right\}$. Now let us prove that the (+)-map is a homomorphism of algebras of signature $\sigma$. Let $G\left(\beta_{1}, \ldots, \beta_{m}\right)=\alpha$, where $G \in \sigma \backslash\left\{\mathrm{J}_{\alpha}^{*} \mid \alpha \in V\right\}$. We shall show that $\hat{G}\left(\beta_{1}^{+}, \ldots, \beta_{m}^{+}\right)=\alpha^{+}$. For this it suffices to prove that $\hat{G}\left(\beta_{1}^{+}, \ldots, \beta_{m}^{+}\right)(\alpha)=1$. In fact, by definition, we have

$$
\left(\hat{G}\left(\beta_{1}^{+}, \ldots, \beta_{m}^{+}\right)\right)(\alpha)=\bigcup_{G\left(\delta_{1}, \ldots, \delta_{m}\right)=\alpha}\left(\bigcap_{j=1}^{m} \beta_{j}^{+}\left(\delta_{j}\right)\right)
$$

Since $G\left(\beta_{1}, \ldots, \beta_{m}\right)=\alpha$ and $\beta_{i}^{+}\left(\beta_{i}\right)=1$ (for $\left.i=1, \ldots, m\right)$, we have $\left(\hat{G}\left(\beta_{1}^{+}, \ldots\right.\right.$, $\left.\left.\beta_{m}^{+}\right)\right)(\alpha)=1$.

Assume that $\mathrm{J}_{\alpha}^{*}(\beta)=1$. Then $\beta=\alpha$ and, therefore, $\hat{\mathrm{J}}_{\alpha}\left(\beta^{+}\right)(1)=\beta^{+}(\alpha)=1$. Thus, $\hat{\mathrm{J}}_{\alpha}\left(\beta^{+}\right)=1^{+}$. Now assume that $\mathrm{J}_{\alpha}^{*}(\beta)=0$. Then $\alpha \neq \beta$ and, by definition, $\hat{\mathbf{J}}_{\alpha}\left(\beta^{+}\right)(0)=-\beta^{+}(\alpha)=-0=1$. Thus, $\hat{\mathbf{J}}_{\alpha}\left(\beta^{+}\right)=0^{+}$.

So we have proved that the (+)-map is an isomorphism from the algebra $\langle V, \sigma\rangle$ onto the subalgebra $\left\langle\left\{\beta^{+} \mid \beta \in V\right\},\{\hat{G} \mid G \in \sigma\}\right\rangle$ of the algebra $B\left(L_{n}\right)$. Moreover, this subalgebra is the $L_{n}$-version of the Boolean subalgebra of the Boolean algebra $B$ 
that is isomorphic to $B_{2}$. Thus, $\langle V, \sigma\rangle$ is isomorphic to $B_{2}\left(L_{n}\right)$. Using the definition of the set $\operatorname{MARK}(\hat{B})$, it is easy to see that $\alpha \in \operatorname{MARK}$ if and only if $\alpha^{+} \in \operatorname{MARK}\left(\hat{B}_{2}\right)$.

Lemma 5.8 Let $h: \mathbf{A F}\left(L_{n}\right) \rightarrow B\left(L_{n}\right)$ be a homomorphism. If a formula $A \in$ $\mathbf{A F}\left(L_{n}\right)$ is $L_{n}$-valid, then $h(A) \in \operatorname{MARK}(\hat{B})$.

Proof: $\quad$ Let $A$ be $L_{n}$-valid. Assume $\mathbf{P V}(A) \cup\left\{p_{0}\right\} \subseteq \wp$. By Corollary 3.15. the formula

$$
\bigwedge_{p \in \wp}(N V)_{p}^{R} \supset\left((\operatorname{mark} A)^{I}\right)^{R}
$$

is a classical tautology.

We set $h_{R}\left(p_{\alpha}\right)=h(p)(\alpha)$, where $\alpha \in V, p \in \mathbf{P V}\left(L_{n}\right)$ and $p_{\alpha} \in \mathbf{P V}(\mathbf{C})$ and then we extend this map to a homomorphism $h_{R}$ of the algebra $\mathbf{A F}(\mathbf{C})$ to the Boolean algebra $B$. Then the homomorphism $h_{R}$ has the value 1 on the formula [2]. Moreover,

$$
h_{R}\left(\bigwedge_{p \in \wp}(N V)_{p}^{R}\right)=\bigcap_{p \in \wp}[[N V]]_{h(p)}=1,
$$

since $h(p) \in \hat{B}$ for $p \in \wp$. Therefore,

$$
h_{R}\left(\left((\operatorname{mark} A)^{I}\right)^{R}\right)=1 .
$$

By our notational stipulations in Section 2, together with Definition 2.12.2 and Definition 3.7, we have

$$
h_{R}\left(\left((\operatorname{mark} A)^{I}\right)^{R}\right)=\bigcup_{\alpha \in \text { MARK }} h_{R}\left(\left(\left(\mathrm{~J}_{\alpha} A\right)^{I}\right)^{R}\right) .
$$

By induction on the logical depth of the formula $A$, it follows that

$$
h_{R}\left(\left(\left(\mathrm{~J}_{\alpha} A\right)^{I}\right)^{R}\right)=h(A)(\alpha) .
$$

$h_{R}$. The inductive step follows from Definitions 2.12.3.7. and 5.1.3.

Identities (3), 44, and 5) imply

$$
\bigcup_{\alpha \in \mathrm{MARK}} h_{R}(A)(\alpha)=1
$$

Thus, $h(A) \in \operatorname{MARK}(\hat{B})$.

Theorem 5.9 A formula A of the language $L_{n}$ is derivable in the calculus $L_{n}$ if and only if for every Boolean algebra $B$ and every homomorphism $h: \mathbf{A F}\left(L_{n}\right) \rightarrow B\left(L_{n}\right)$ it is the case that $h(A) \in \operatorname{MARK}(\hat{B})$.

Proof: Using Theorems 3.5. 3.16, and Lemma 5.7.2, one argues as in the proof of Lemma 5.8.

Definition 5.10 We call the algebra $\left\langle M,\left\{F^{\circ} \mid F \in \sigma\right\}\right\rangle$, where $F^{\circ}$ is an operation with the same number of arguments as $F$, an $L_{n}$-algebra if for any $a, b, c \in M$ and $\alpha, \beta \in V$ the following conditions hold: 
1. Axioms of exterior elements (Ex):

(EX1) $\mathrm{J}_{\alpha}^{\circ} a \vee^{\circ} \mathbf{J}_{\alpha}^{\circ} a=\mathrm{J}_{\alpha}^{\circ} a$,

(EX2) $\mathrm{J}_{\alpha}^{\circ} a \vee^{\circ} \mathbf{J}_{\beta}^{\circ} b=\mathrm{J}_{\beta}^{\circ} b \vee^{\circ} \mathbf{J}_{\alpha}^{\circ} a$,

(EX3) $\left(\mathbf{J}_{\alpha}^{\circ} a \vee^{\circ} \mathbf{J}_{\beta}^{\circ} b\right) \vee^{\circ} \mathbf{J}_{\gamma}^{\circ} c=\mathbf{J}_{\alpha}^{\circ} a \vee^{\circ}\left(\mathbf{J}_{\beta}^{\circ} b \vee^{\circ} \mathbf{J}_{\gamma}^{\circ} c\right)$,

(EX4) $\mathbf{J}_{\alpha}^{\circ} a \wedge^{\circ}\left(\mathbf{J}_{\beta}^{\circ} b \vee^{\circ} \mathbf{J}_{\gamma}^{\circ} c\right)=\left(\mathbf{J}_{\alpha}^{\circ} a \wedge^{\circ} \mathbf{J}_{\beta}^{\circ} b\right) \vee^{\circ}\left(\mathbf{J}_{\alpha}^{\circ} a \wedge^{\circ} \mathbf{J}_{\gamma}^{\circ} c\right)$,

(EX5) $\neg^{\circ} \neg^{\circ} \mathrm{J}_{\alpha}^{\circ} a=\mathrm{J}_{\alpha}^{\circ} a$,

(EX6) $\neg^{\circ}\left(\mathbf{J}_{\alpha}^{\circ} a \vee^{\circ} \mathbf{J}_{\beta}^{\circ} b\right)=\neg^{\circ} \mathbf{J}_{\alpha}^{\circ} a \wedge^{\circ} \neg^{\circ} \mathbf{J}_{\beta}^{\circ} b$,

(EX7) $\mathrm{J}_{\alpha}^{\circ} a \vee^{\circ}\left(\mathbf{J}_{\beta}^{\circ} b \wedge^{\circ} \neg^{\circ} \mathbf{J}_{\beta}^{\circ} b\right)=\mathbf{J}_{\alpha}^{\circ} a$,

(EX8) $\mathbf{J}_{\alpha}^{\circ} a \supset^{\circ} \mathbf{J}_{\beta}^{\circ} b=\neg^{\circ} \mathbf{J}_{\alpha}^{\circ} a \vee^{\circ} \mathbf{J}_{\beta}^{\circ} b$,

2. Axioms of $n$-valuedness ( $n$-Val):

$\left(n-\mathrm{Val}_{\alpha}\right)$

$$
\mathrm{J}_{\alpha}^{\circ} a=\bigwedge_{\beta \neq \alpha}^{\circ} \neg^{\circ} \mathbf{J}_{\beta}^{\circ} a,
$$

(for each $\alpha \in V$ ), where

$$
\bigwedge_{i \in I}^{\circ} a_{i}= \begin{cases}\mathbf{J}_{1}^{\circ} b \vee^{\circ} \neg^{\circ} \mathbf{J}_{1}^{\circ} b & \text { if } I=\varnothing, \\ \left(a_{i_{1}} \wedge^{\circ} \ldots\left(\ldots \wedge^{\circ} a_{i_{k}}\right) \ldots\right) & \text { if } I=\left\{i_{1}, \ldots, i_{k}\right\} .\end{cases}
$$

3. Connective Axioms ( $\mathrm{Cn})$ :

(Cn-F)

$$
\mathbf{J}_{\alpha}^{\circ}\left(F^{\circ}\left(a_{1}, \ldots, a_{m}\right)\right)=\bigvee_{F\left(\beta_{1}, \ldots, \beta_{m}\right)=\alpha}^{\circ} \bigwedge_{j=1}^{m} \mathbf{J}_{\beta_{j}}^{\circ} a_{j},
$$

where $F \in \sigma \backslash\left\{\mathrm{J}_{\alpha}^{*} \mid \alpha \in V\right\}$ and

$$
\bigvee_{i \in I}^{\circ} a_{i}= \begin{cases}\mathbf{J}_{1}^{\circ} b \wedge^{\circ} \neg^{\circ} \mathbf{J}_{1}^{\circ} b & \text { if } I=\varnothing, \\ \left(a_{i_{1}} \vee^{\circ} \ldots\left(\ldots \vee^{\circ} a_{i_{k}}\right) \ldots\right) & \text { if } I=\left\{i_{1}, \ldots, i_{k}\right\} .\end{cases}
$$

$\left(\mathbf{C n} \mathrm{J}_{0}\right) \quad J_{0}^{\circ} J_{\beta}^{\circ} a=\neg^{\circ} \mathbf{J}_{\beta}^{\circ} a$,

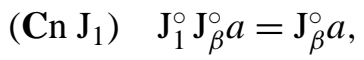

$\left(\mathbf{C n} \mathbf{J}_{\alpha}\right) \quad \mathbf{J}_{\alpha}^{\circ} \mathbf{J}_{\beta}^{\circ} a=\mathbf{J}_{1}^{\circ} a \wedge^{\circ} \neg^{\circ} \mathbf{J}_{1}^{\circ} a$ for $\alpha \notin\{0,1\}$.

4. Axioms of closure (C1):

$\left(\mathbf{C} 1^{*}\right) \quad \mathbf{J}_{\alpha}^{\circ} a *^{\circ} \mathbf{J}_{\beta}^{\circ} b=\mathbf{J}_{1}^{\circ}\left(\mathbf{J}_{\alpha}^{\circ} a *^{\circ} \mathbf{J}_{\beta}^{\circ} b\right)$, where $*^{\circ}$ denotes one of the operations $\wedge^{\circ}, \vee^{\circ}$ or $\supset^{\circ}$,

$(\mathbf{C} 1 \neg) \quad \neg^{\circ} \mathbf{J}_{\alpha}^{\circ} b=\mathbf{J}_{1}^{\circ}\left(\neg^{\circ} \mathbf{J}_{\alpha}^{\circ} b\right)$,

5. Quasi-identity (QI):

$$
\underset{\alpha \in V}{\mathbf{\&}}\left(\mathbf{J}_{\alpha}^{\circ} a=\mathbf{J}_{\alpha}^{\circ} b\right) \Longrightarrow a=b .
$$

Lemma 5.11 Let $\vdash_{L_{n}} X \equiv Y$. Then for every Boolean algebra $B$ and every homomorphism $h: \mathbf{A F}\left(L_{n}\right) \rightarrow B\left(L_{n}\right)$ it is true that $h(X)=h(Y)$. 
Proof: Let $\vdash_{L_{n}} X \equiv Y$. Then, by Proposition 2.6 and axioms $\left(\mathbf{C J}_{0}\right),\left(\mathbf{C J}_{1}\right)$, and $\left(\mathbf{C J}_{\alpha}\right)$ of the calculus $L_{n}$, we have $\vdash_{L_{n}} \mathrm{~J}_{\alpha} X \equiv \mathrm{J}_{\alpha} Y$ for each $\alpha \in V$. Therefore, by Lemma 2.13 and Proposition 2.6. $\vdash{ }_{L_{n}}\left(\mathrm{~J}_{\alpha} X\right)^{I} \equiv\left(\mathrm{J}_{\alpha} Y\right)^{I}$. Then, by Theorem 3.5 and Lemma 3.14, for each $\alpha \in V$, the formula

$$
\bigwedge_{p \in \wp}(N V)_{p}^{R} \supset\left(\left(\left(\mathrm{J}_{\alpha} X\right)^{I}\right)^{R} \equiv\left(\left(\mathrm{J}_{\alpha} Y\right)^{I}\right)^{R}\right)
$$

will be a classical tautology, where $\left\{p_{0}\right\} \cup \mathbf{P V}(X) \cup \mathbf{P V}(Y) \subseteq \wp$. If $h: \mathbf{A F}\left(L_{n}\right) \rightarrow$ $B\left(L_{n}\right)$ is a homomorphism, then (in a manner following that employed in the proof of Lemma 5.8) it is possible to define the homomorphism $h_{R}: \mathbf{A F}(\mathbf{C}) \rightarrow B$ of these algebras in such way that the following conditions hold:

$$
\begin{array}{r}
h_{R}\left(\bigwedge_{p \in \wp}(N V)_{p}^{R}\right)=1, \\
h_{R}\left(\left(\left(\mathrm{~J}_{\alpha} X\right)^{I}\right)^{R}\right)=h(X)(\alpha), \\
h_{R}\left(\left(\left(\mathrm{~J}_{\alpha} Y\right)^{I}\right)^{R}\right)=h(Y)(\alpha) .
\end{array}
$$

From identity (7) and the fact that the value of the homomorphism $h_{R}$ on the formula [6] is equal to 1 , it follows that

$$
h_{R}\left(\left(\left(\mathrm{~J}_{\alpha} X\right)^{I}\right)^{R} \equiv\left(\left(\mathrm{J}_{\alpha} Y\right)^{I}\right)^{R}\right)=1,
$$

for all $\alpha \in V$. And by identities (8) and 9, it follows that $h(X)=h(Y)$.

Theorem 5.12 For any Boolean algebra $B$ its $L_{n}$-version $B\left(L_{n}\right)$ is an $L_{n}$-algebra.

Proof: $\quad$ Let $a, a_{1}, \ldots, a_{m}, b \in \hat{B}$ and $p, p_{1}, \ldots, p_{m}, q \in \mathbf{P V}\left(L_{n}\right)$, where all these propositional variables are different. We set $h(p)=a, h(q)=b, h\left(p_{i}\right)=a_{i}$ for $i=$ $1, \ldots, m$, and extend this map to an arbitrary homomorphism $h: \mathbf{A F}\left(L_{n}\right) \rightarrow B\left(L_{n}\right)$ of these algebras.

By Proposition 2.6, we have $\vdash_{L_{n}} \neg \neg \mathrm{J}_{\alpha} p \equiv \mathrm{J}_{\alpha} p$. Then, by Lemma 5.11, we have $h\left(\neg \neg \mathrm{J}_{\alpha} p\right)=h\left(\mathrm{~J}_{\alpha} p\right)$ and, therefore, $\hat{\neg} \hat{\neg} \hat{\mathbf{J}}_{\alpha} a=\hat{\mathbf{J}}_{\alpha} a$. This means that the axiom (Ex5) of $L_{n}$-algebra holds. The other axioms of the group (Ex) hold for analagous reasons. For the cases of the axioms $(\mathbf{C n}),(\mathbf{C l}),\left(n-\mathrm{Val}_{\alpha}\right)$ we need to appeal to the axioms (Con), $\left(\mathbf{C J}_{\alpha}\right)$, and $\left(N V_{\alpha}\right)$ of the calculus $L_{n}$, respectively.

Let us now show that the quasi-identity (QI) is true in the algebra $B\left(L_{n}\right)$. Let $\hat{\mathrm{J}}_{\alpha} a=\hat{\mathbf{J}}_{\alpha} b$ and $a \neq b$. In order to obtain a contradiction, suppose that $a(\alpha) \neq b(\alpha)$ for some $\alpha \in V$. Then, by Definition 5.1. 3, we have

$$
\left(\hat{\mathbf{J}}_{\alpha} a\right)(1)=a(\alpha) \neq b(\alpha)=\left(\hat{\mathbf{J}}_{\alpha} b\right)(1),
$$

a contradiction.

Definition 5.13 Let $\mathcal{M}=\left\langle M,\left\{F^{\circ} \mid F \in \sigma\right\}\right\rangle$ be an $L_{n}$-algebra. We call the set $\mathbf{E}(M)=\left\{\mathrm{J}_{\alpha}^{\circ} a \mid a \in M, \alpha \in V\right\}$ the set of external elements of the $L_{n}$-algebra $\mathcal{M}$. Moreover, we define the set

$$
\operatorname{MARK}[M]=\left\{a \in M \mid \bigvee_{\alpha \in \operatorname{MARK}}^{\circ} \mathbf{J}_{\alpha}^{\circ} a=\mathbf{J}_{1}^{\circ} a \vee^{\circ} \neg^{\circ} \mathbf{J}_{1}^{\circ} a\right\} .
$$


Theorem 5.14 (First Representation Theorem) Let $\mathcal{M}=\left\langle M,\left\{F^{\circ} \mid F \in \sigma\right\}\right\rangle$ be an $L_{n}$-algebra. Then there exists a monomorphism of $L_{n}$-algebras $g: \mathcal{M} \rightarrow B\left(L_{n}\right)$ for some Boolean algebra $B$.

Proof: By axioms $(\mathbf{C} 1)$, the set $\mathbf{E}(\mathrm{M})$ is closed under the operations $\wedge^{\circ}, \vee^{\circ}, \supset^{\circ}$, and $\neg^{\circ}$. By axioms (Ex), the algebra $B=\left\langle\mathbf{E}(M), \wedge^{\circ}, \vee^{\circ}, \neg^{\circ}\right\rangle$ is a Boolean algebra and $a \supset^{\circ} b=\neg^{\circ} a \vee^{\circ} b$ for every $a, b \in \mathbf{E}(M)$ (see for example Huntington's theorem in Chapter 2 of Birkhoff [8]). We define the map $g: M \rightarrow(\mathbf{E}(M))^{V}$ by the rule $g(a)(\alpha)=\mathrm{J}_{\alpha}^{\circ} a$, for every $\alpha \in V$ and $a \in M$. By the axioms ( $n$-Val), we have $g(a) \in(\mathbf{E}(\hat{M}))$ (see Definition 5.1). By the quasi-identity (QI), the map $g$ is an injection. Moreover, by the axioms $(\mathbf{C n})$, this map $g$ is a homomorphism of $L_{n}$-algebras.

Lemma 5.15 Let $B\left(L_{n}\right)=\langle\hat{B},\{\hat{F} \mid F \in \sigma\}\rangle$ is the $L_{n}$-version of a Boolean algebra B. Then $\operatorname{MARK}(\hat{B})=\operatorname{MARK}[\hat{B}]$ (see Definitions 5.1 and 5.13].

Proof: $\quad$ It is easy to see that $\mathbf{E}(\hat{B})=\{\hat{a} \mid a \in B\}$, since for $x \in \hat{B}$ and $\beta \in V$ we have $\hat{\mathbf{J}}_{\beta} x=x(\hat{\beta})$ and $\hat{a}=\hat{\mathbf{J}}_{1} \hat{a}$ (see Definition 5.1).

We set $\hat{a} \ll \hat{b}$ if and only if $\hat{a} \hat{\wedge} \hat{b}=\hat{a}$. Then $\langle\mathbf{E}(\hat{B}), \ll\rangle$ is a Boolean algebra (see the proof of Theorem 5.14). Let us show that the map $f: B \rightarrow \mathbf{E}(\hat{B})$, defined by the rule $f(a)=\hat{a}$ for all $a \in B$, is an isomorphism of Boolean algebras. Obviously, the map $f$ is a bijection. Thus, it suffices to show that $f$ preserves the partial orders $\leq$ and $\ll$ of our Boolean algebras.

Let $a \leq b$ for $a, b \in B$. Let us consider the following identity:

$$
(\hat{a} \hat{\wedge} \hat{b})(1)=\bigcup_{\alpha \wedge * \beta=1}(\hat{a}(\alpha) \cap \hat{b}(\beta))
$$

It is clear that if $\alpha \notin\{0,1\}$ or $\beta \notin\{0,1\}$, then $\hat{a}(\alpha) \cap \hat{b}(\beta)=0$. Therefore, a summand of the Boolean sum on the right hand side of the above equality is not equal to 0 if and only if $\alpha, \beta \in\{0,1\}$. But $\alpha \wedge^{*} \beta=1$ and, therefore, $\alpha=\beta=1$. Thus,

$$
(\hat{a} \hat{\wedge} \hat{b})(1)=\hat{a}(1) \cap \hat{b}(1)=a \cap b=a .
$$

Therefore, $\hat{a} \hat{\wedge} \hat{b}=\hat{a}$; stated otherwise, $\hat{a} \ll \hat{b}$. Moreover, it is clear that $\hat{a} \ll \hat{b}$ implies $a \leq b$ for every $a, b \in B$. Let us define the map $h: \mathbf{E}(\hat{B}) \rightarrow B$ by the rule $h\left(\hat{\mathbf{J}}_{\alpha} x\right)=$ $h(x \hat{(\alpha)})=x(\alpha)$, for all $\alpha \in V$ and $x \in \hat{B}$. This map $h$ is the inverse map for the isomorphism $f$ and, therefore, $h$ is also an isomorphism of Boolean algebras. Thus, for every $x \in \hat{B}$, it is true that

$$
\widehat{\bigvee}_{\alpha \in \mathrm{MARK}} \hat{\mathbf{J}}_{\alpha} x=\hat{\mathbf{J}}_{1} x \hat{\vee} \hat{\neg} \hat{\mathbf{J}}_{1} x
$$

if and only if

$$
\bigcup_{\alpha \in \operatorname{MARK}} x(\alpha)=1
$$

So $\operatorname{MARK}[\hat{B}]=\operatorname{MARK}(\hat{B})$.

Corollary 5.16 The algebra $\langle V, \sigma\rangle$ of the logic $L_{n}$ is an $L_{n}$-algebra such that $\operatorname{MARK}[V]=$ MARK. 
Proof: From Lemma 5.7.2 together with Theorem 5.12 and Lemma 5.15.

Lemma 5.17 Let $\mathcal{M}=\left\langle M,\left\{F^{\circ} \mid F \in \sigma\right\}\right\rangle$ be an $L_{n}$-algebra and $h: \mathbf{A F}\left(L_{n}\right) \rightarrow$ $\mathcal{M}$ be a homomorphism. If $A$ is an $L_{n}$-valid formula of the logic $L_{n}$, then $h(A) \in$ MARK $[M]$.

Proof: In fact, the algebra $B=\left\langle\mathbf{E}(M), \wedge^{\circ}, \vee^{\circ}, \neg^{\circ}\right\rangle$ is a Boolean algebra, and the map $g: M \rightarrow \mathbf{E}(\hat{M})$, defined for all $a \in M, \alpha \in V$ by the rule $g(a)(\alpha)=\mathrm{J}_{\alpha}^{\circ} a$, is a monomorphism from the algebra $\mathcal{M}$ to the algebra $B\left(L_{n}\right)$ (see the proof of Theorem 5.14. Moreover, it is clear that $a \in \operatorname{MARK}[M]$ if and only if $g(a) \in \operatorname{MARK}(\hat{B})$.

Let us identify the algebra $\mathcal{M}$ with its image $g(\mathcal{M})$ in the algebra $B\left(L_{n}\right)$. Then $h$ will be the homomorphism from the algebra $\mathbf{A F}\left(L_{n}\right)$ to the algebra $B\left(L_{n}\right)$. Therefore, our lemma follows from Lemmas 5.8 and 5.15.

Theorem 5.18 The formula A of the logic $L_{n}$ is $L_{n}$-derivable if and only if for every $L_{n}$-algebra $\mathcal{M}=\left\langle M,\left\{F^{\circ} \mid F \in \sigma\right\}\right\rangle$ and every homomorphism $h: \mathbf{A F}\left(L_{n}\right) \rightarrow \mathcal{M}$ it is true that $h(A) \in \mathbf{M A R K}[M]$.

Proof: Similar to the proof of Theorem 5.9.

Definition 5.19 Let $\mathcal{D}^{I}$ be a Cartesian power of the algebra $\mathcal{D}=\langle D, \tau\rangle$, and $K$ be any set. If $f: K \rightarrow D^{I}$ is a map, then we shall denote by $f_{i}$, where $i \in I$, the map defined by the rule $f_{i}(a)=f(a)(i)$, for all $a \in K$. As usual, we denote by $B_{2}$ the two-element Boolean algebra.

Theorem 5.20 (Second Representation Theorem) Every $L_{n}$-algebra is isomorphically embeddable into a Cartesian power of the algebra $\langle V, \sigma\rangle$ of the logic $L_{n}$.

Proof: Let $\mathcal{M}=\left\langle M,\left\{F^{\circ} \mid F \in \sigma\right\}\right\rangle$ be an $L_{n}$-algebra. By Lemma 5.7.2, it is possible to identify the algebra $\langle V, \sigma\rangle$ with the algebra $B_{2}\left(L_{n}\right)$. Now by Theorem 5.14. there exist a Boolean algebra $B$ and a monomorphism of $L_{n}$-algebras $h: \mathcal{M} \rightarrow B\left(L_{n}\right)$. And by the Birkhoff-Stone Theorem (see Birkhoff [8], Chapter 8, Section 8), for some set $I$ there exists a monomorphism $g: B \rightarrow B_{2}^{I}$. Let us prove that there exists a monomorphism $G: \mathcal{M} \rightarrow\left(B_{2}\left(L_{n}\right)\right)^{I}$. In order to define $G$, it suffices to define its projections $G_{i}$ for all $i \in I$. (Note that for every $a \in M, G_{i}(a)$ is a map from $V$ to $\{0,1\}$.) We define $G_{i}$ by the following rule:

$$
G_{i}(a)(\alpha)=g_{i}(h(a)(\alpha))
$$

for $\alpha \in V$ and $a \in M$.

Now let us show that $G_{i}(a) \in \hat{B}_{2}$ for all $a \in M$. It suffices to prove the following identities:

$$
\begin{aligned}
\bigcup_{\alpha \in V} G_{i}(a)(\alpha) & =1, \\
\bigcup_{\beta \neq \alpha \in V}\left(G_{i}(a)(\alpha) \cap G_{i}(a)(\beta)\right) & =0 .
\end{aligned}
$$


Since $h(M) \subseteq \hat{B}$, we have

$$
\begin{aligned}
\bigcup_{\alpha \in V} h(a)(\alpha) & =1 \in B, \\
\bigcup_{\beta \neq \alpha \in V}(h(a)(\alpha) \cap h(a)(\beta)) & =0 \in B .
\end{aligned}
$$

Therefore, we have the two identities

$$
\begin{aligned}
\bigcup_{\alpha \in V} g_{i}(h(a)(\alpha)) & =1, \\
\bigcup_{\beta \neq \alpha \in V} g_{i}(h(a)(\alpha) \cap h(a)(\beta)) & =0 .
\end{aligned}
$$

In light of (10), the identities 15 and (16) imply (11) and (12). Now let us show that the map $G_{i}: \mathcal{M} \rightarrow B_{2}\left(L_{n}\right)$ is a homomorphism of $L_{n}$-algebras. In order to prove that

$$
G_{i}\left(F^{\circ}\left(a_{1}, \ldots, a_{r}\right)\right)=\hat{F}\left(G_{i}\left(a_{1}\right), \ldots, G_{i}\left(a_{r}\right)\right),
$$

where $F \in \sigma \backslash\left\{\mathrm{J}_{\alpha}^{*} \mid \alpha \in V\right\}$ and $a_{1}, \ldots, a_{r} \in M$, it suffices to prove that for every $\alpha \in V$ it is true that

$$
G_{i}\left(F^{\circ}\left(a_{1}, \ldots, a_{r}\right)\right)(\alpha)=\hat{F}\left(G_{i}\left(a_{1}\right), \ldots, G_{i}\left(a_{r}\right)\right)(\alpha) .
$$

Let us consider the following chain of identities:

$$
\begin{aligned}
G_{i}\left(F^{\circ}\left(a_{1}, \ldots, a_{r}\right)\right)(\alpha) & =g_{i}\left(h\left(F^{\circ}\left(a_{1}, \ldots, a_{r}\right)\right)(\alpha)\right) \\
& =g_{i}\left(\hat{F}\left(h\left(a_{1}\right), \ldots, h\left(a_{r}\right)\right)(\alpha)\right) \\
& =g_{i}\left(\bigcup_{F\left(\beta_{1}, \ldots, \beta_{r}\right)=\alpha}\left(\bigcap_{j=1}^{r} h\left(a_{j}\right)\left(\beta_{j}\right)\right)\right) \\
& =\bigcup_{F\left(\beta_{1}, \ldots, \beta_{r}\right)=\alpha}\left(\bigcap_{j=1}^{r} g_{i}\left(h\left(a_{j}\right)\left(\beta_{j}\right)\right)\right) \\
& =\bigcup_{F\left(\beta_{1}, \ldots, \beta_{r}\right)=\alpha}\left(\bigcap_{j=1}^{r} G_{i}\left(a_{j}\right)\left(\beta_{j}\right)\right) \\
& =\hat{F}\left(G_{i}\left(a_{1}\right), \ldots, G_{i}\left(a_{r}\right)\right)(\alpha) .
\end{aligned}
$$

The first and the fifth identities are true by 10 , the third by Definition 5.1. 3 , and the sixth by Definition 5.1.3. The proof that

$$
\left(G_{i}\left(\mathrm{~J}_{\beta}^{\circ} a\right)\right)(\alpha)=\left(\hat{\mathrm{J}}_{\beta} G_{i}(a)\right)(\alpha)
$$

is true for every $\alpha \in V$ and $a \in M$, is similar to the proof of 117 . So we have proved that $G_{i}$ is a homomorphism for every $i \in I$.

Let us define the map $G: \mathcal{M} \rightarrow\left(\hat{B}_{2}\right)^{I}$ by the rule $G(a)(i)=G_{i}(a)$, for all $a \in$ $M$. Then $G: \mathcal{M} \rightarrow\left(B_{2}\left(L_{n}\right)\right)^{I}$ is a homomorphism of $L_{n}$-algebras. Let us show that $G$ is a monomorphism. Indeed, let $a, b \in M$ and $a \neq b$. Then $h(a) \neq h(b)$. Therefore, there exists $\alpha \in V$ such that $h(a)(\alpha) \neq h(b)(\alpha)$. Then $g(h(a)(\alpha)) \neq g(h(b)(\alpha))$. Thus there is an $i \in I$ such that $g_{i}(h(a)(\alpha)) \neq g_{i}(h(b)(\alpha))$. By 10], it follows that $G_{i}(a)(\alpha) \neq G_{i}(b)(\alpha)$. Thus, $G_{i}(a) \neq G_{i}(b)$, whence $G(a) \neq G(b)$. 
Corollary 5.21 Every $L_{n}$-algebra is isomorphic to a subdirect product of subalgebras of the algebra $\langle V, \sigma\rangle$ of the logic $L_{n}$.

Corollary 5.22 If an $L_{n}$-algebra $\mathcal{M}$ is subdirectly irreducible (i.e., it cannot be decomposed into a proper product in the quasi-variety of all $L_{n}$-algebras) then $\mathcal{M}$ is isomorphic to a subalgebra of $\langle V, \sigma\rangle$.

\section{REFERENCES}

[1] Anshakov, O. M., Intuitionistic versions of finite-valued logics, Doctoral Dissertation, MGPI, Moscow, 1984 (in Russian).

[2] Anshakov, O. M., and S. V. Rychkov, "On multivalued logical calculi," Soviet Math. Dokl., vol. 25 (1982), pp. 599-602.Zbl 0507.03011 1.1.1.1.4.

[3] Anshakov, O. M., and S. V. Rychkov, "Many-valued logical calculi," Semiotika i Informatika, vol. 19 (1982), pp. 90-117 (in Russian).Zbl 0501.03012 MR 84e:03031 1. I1. 4

[4] Anshakov, O. M., and S. V. Rychkov, "Towards the theory of many-valued logical calculi," Semiotika i Informatika, vol. 23 (1984), pp. 76-108 (in Russian). 1.1.1.4.

[5] Anshakov, O. M., and S. V. Rychkov, "On the axiomatization of finite-valued logical calculi," Math. USSR Sbornik, vol. 51 (1985), pp. 473-491.ZZbl 0566.03010 MR 85i:03065 1.11.11.11.4

[6] Anshakov, O. M., and S. V. Rychkov, "Many-valued logics and their algebras," Russian Mathematical Surveys, vol. 45 (1990), pp. 139-140.Zbl 0725.03008|MR 92i:03019]

[7] Anshakov, O. M., V. K. Finn, and D. P. Skvortsov, "On axiomatization of many-valued logics associated with formalization of plausible reasoning," Studia Logica, vol. 48 (1990), pp. 423-447. MR 91m:03023 1,1

[8] Birkhoff, G., Lattice theory, American Mathematical Society, Providence, 1967. Zbl 0153.02501|MR 37:2638 5,5

[9] Bochvar, D. A., "On a three-valued logical calculus and its application to the analysis of the paradoxes of the classical extended functional calculus," Math. USSR Sbornik, vol. 4 (1938), pp. 287-308 (in Russian; English translation in: History and Philosophy of Logic, vol. 2 (1981)). Zbl 0512.03004/MR 83f:03022 1,11,4

[10] Bochvar D. A., and V. K. Finn, "Some additions to articles on many-valued logics," pp. 265-325 in Investigations on Set Theory and Non-Classical Logics, Nauka, Moscow, 1976 (in Russian).

[11] Carnielli, W. A., "Systematization of finite-valued logics through the method of tableaux," The Journal of Symbolic Logic, vol. 52 (1987), pp. 473-493.Zbl 0633.03008 4

[12] Carnielli, W. A., "On sequents and tableaux for many-valued logics," The Journal of Non-Classical Logic, vol. 8 (1991), pp. 59-76. Zbl 0774.03006 MR 94b:03046 4

[13] Chang, C. C., "Algebraic analysis of many-valued logics," Transactions of the American Mathematical Society, vol. 88 (1958), pp. 467-490. Zbl 0084.00704 MR 20:821 1

[14] Chang, C. C., "A new proof of the completeness of the Łukasiewicz axioms," Transactions of the American Mathematical Society, vol. 93 (1959), pp. 74-80.Zbl 0093.01104 MR 23:A58 
[15] Cignoli, R., "An algebraic approach to elementary theories based on $n$-valued Łukasiewicz logics," Zeitschrift für mathematische Logik und Grundlagen der Mathematik, vol. 30 (1984), pp. 87-96.ZZbl 0551.03037 MR 85i:03191 1

[16] Cleave, J. T., "The notion of logical consequence in the logic of inexact predicates," Zeitschrift für mathematische Logik und Grundlagen der Mathematik, vol. 20 (1974), pp. 307-324. Zbl 0299.02015 MR 51:10028 1

[17] Ebbinghaus, H.-D., "Über eine Prädikatenlogik mit partiell definierten Prädikaten und Funktionen," Archiv für mathematische Logik und Grundlagenforschriften, vol. 12 (1969), pp. 39-53.Zbl 0182.31502|MR 40:7092 1.4

[18] Figallo, A., "I $\Delta_{3}$-algebras," Reports of Mathematical Logic, vol. 24 (1990), pp. 3-16. MR 92c:03071 1

[19] Finn, V. K., "On the functional properties and axiomatization of D. A. Bochvar's threevalued logic," pp. 22-23 in 4th International Congress of Logic, Methodology and Philosophy of Science, Bucharest, 1971.

[20] Finn, V. K., "Axiomatization of some three-valued propositional calculi and their algebras," pp. 398-438 in Philosophy in the Modern World, Philosophy and Logic, Nauka, Moscow, 1974 (in Russian). MR 58:27304 1

[21] Finn, V. K., O. M. Anshakov, R. Grigolia, and M. I. Zabezhailo, "Many-valued logics as fragments of formalized semantics," Acta Philosophica Fennica, vol. 35 (1982), pp. 239-271. Zbl 0521.03011|MR 86f:03040 1,1,11

[22] Finn, V., and R. Grigolia, "Bochvar's algebras and their corresponding propositional calculi," Bulletin of the Section of Logic, vol. 9 (1980), pp. 39-45.

[23] Girard, J.-Y., "Three-valued logics and cut-elimination: the actual meaning of Takeuti's conjecture," Dissertationes Mathematica, vol. 136 (1976), pp. 1-49. MR 56:5235 1

[24] Goddard, L., and R. Routley, The Logic of Significance and Context, vol. 1, Scottish Academic Press, Edinburgh, 1973. Zbl 0302.02004 MR 58:5052 1,4

[25] Grigolia, R., "Free Łukasiewicz algebras," pp. 64-74 in Methods for research in logic, Metsnireba, Tbilisi, 1987 (in Russian).

[26] Grigolia, R., "Algebraic analysis of Łukasiewicz-Tarski's $n$-valued logical systems," pp. 81-92 in Selected Papers on Łukasiewicz Sentential Calculi, edited by G. Malinowski and R. Wójcicki, Ossolineum, Wrocław, 1977. MR 58:21437 1

[27] Grigolia, R., and V. K. Finn, "Bochvar's algebras and their corresponding propositional calculi," pp. 345-372 in Studies in Non-classical Logics and Set Theory, edited by A. I. Michailov, Nauka, Moscow, 1979 (in Russian). Zbl 0441.03023 MR 81f:03031 1,1,1,1]

[28] Guzman, F., "The implications in conditional logic," Lecture Notes in Computer Science, vol. 425 (1990), pp. 139-159. Zbl 0783.03008 MR 92e:03028 1

[29] Guzman, F., and C. Squier, "The algebras of conditional logics," Algebra Universalis, vol. 29 (1990), pp. 88-110. MR 90m:03107 1,1

[30] Hähnle, R.,Tableaux based methods in many-valued automated deduction, Oxford University Press, Oxford, 1993.

[31] Hallden, S., "The logic of nonsense," Arsskrift, Heft 1, Uppsala University, 1949. $\mathrm{Zbl} 0040.29201 \mathrm{D}$ 
[32] Hoogewijs, A., "On a formalization of the non-definedness notion," Zeitschrift für mathematische Logik und Grundlagen der Mathematik, vol. 25 (1979), pp. 213-217. Zbl 0415.03019||MR 80f:03017,1

[33] Komori, Y., “Super Łukasiewicz propositional logics," Nayoya Mathematical Journal, vol. 84 (1981), pp. 119-133. Zbl 0482.03007|MR 83i:03041 1

[34] Komori, Y., "Super Łukasiewicz implicational logics," Nayoya Mathematical Journal, vol. 72 (1978), pp. 127-133. Zbl 0363.02015 MR 80d:03021 1

[35] Łukasiewicz, J., and A. Tarski, "Investigations into the sentential calculus," Chapter IV in Logic, Semantics, Metamathematics, by A. Tarski, Clarendon Press, Oxford, 1956. 1.4

[36] Moisil, G. C., "Notes sur les logiques nonchrysippiennes," Ann. Sci. Univ. Iassy, vol. 27 (1941), 86-98.

[37] Mundici, D., "Satisfiability in many-valued sentential logic is NP-complete," Theoretical Computer Science, vol. 52 (1987), pp. 145-153.Zbl 0639.03042 MR 89a:68076 1.

[38] Mundici, D., "The derivative of truth in Łukasiewicz sentential calculi," Contemporary Mathematics, vol. 69 (1988), pp. 209-277. Zbl 0648.03011MR 89b:03045 1

[39] Mundici, D., "The $C^{*}$-algebras and three-valued logics," pp. 61-77 in Proceedings of the Logic Colloquium '88. Studies in Logic and the Foundations of Mathematics, North Holland, 1989. MR 90j:03039 1

[40] D'Ottaviano, I., "The model extension theorem for J3 theories," Lecture Notes in Mathematics, vol. 1130 (1985), pp. 157-173. MR 87b:03048 1

[41] D'Ottaviano, I., "The completeness and compactness of a 3-valued first-order logic," Revista Colombiana de Matematica, vol. 19 (1985), pp. 77-94.Zbl 0614.03020 MR 88a:03055 1

[42] D'Ottaviano, I., "Definability and quantifier elimination for J3," Studia Logica, vol. 46 (1987), pp. 37-54.Zbl0633.03007/MR 88m:03037

[43] Płonka, J., "On distributive quasi-lattices," Fundamenta Mathematica, vol. 60 (1967), pp. 191-200. Zbl 0154.00709MR 36:85

[44] Post, E., "Introduction to a general theory of elementary propositions," American Journal of Mathematics, vol. 43 (1921), pp. 63-185.

[45] Rasiowa, H., An algebraic approach to non-classical logics, North-Holland, Amsterdam, 1974. Zbl 0299.02069|MR 56:5285 1

[46] Rose, A., and J. Rosser, "Fragments of many-valued statement calculi," Transactions of the American Mathematical Society, vol. 87 (1958), pp. 1-53.Zbl 0085.24303 MR 20:818 1,4

[47] Rosenberg, I. G., "Completeness properties of multi-valued logic algebras," pp. 144186 in Computer Science and multiple-valued logic, edited by D. C. Rine, NorthHolland, Amsterdam, 1977.

[48] Rosser, J. B., and A. R. Turquette, Many-valued logics, North-Holland, Amsterdam, 1951. Zbl 0047.01503 MR 14,526a 1

[49] Rousseau, G., 'Sequents in many-valued logic I,' Fundamenta Mathematica, vol. 60 (1967), pp. 23-33. Erratum: Fundamenta Mathematica, vol. 61 (1967-68), p. 313. Zbl 0154.25504IMR 35:1451 [.II.4. 
[50] Rousseau, G., "Sequents in many-valued logic II," Fundamenta Mathematica vol. 67 (1970), pp. 125-131.Zbl 0194.30703|MR 41:6671 1

[51] Rousseau, G., "Post algebras and pseudo-Post algebras," Fundamenta Mathematica vol. 67 (1970), pp. 133-145.Zbl 0198.01904MR 42:159 1

[52] Rychkov, S. V., "On the Completeness Theorem for many-valued structures." Russian Mathematical Surveys, vol. 46 (1991), pp. 227-228.Zbl 0811.03017|MR 1164203 1

[53] Segerberg, K., “A contribution to nonsense logics," Theoria, vol. 31 (1965), pp. 199217.

[54] Smullyan, R., First-order logic, Springer-Verlag, Berlin, 1968.Zbl 0172.28901 MR 39:5311 1

[55] Suchon, W., "La méthode de Smullyan de construire le calcul $n$-valent de Łukasiewicz avec implication et negation," Reports Mathematical Logic, vol. 2 (1974) pp. 37-42. $\mathrm{Zbl}$ 0284.02007|lMR 54:2419 1

[56] Surma, S. J., "An algorithm for axiomatizing every finite logic," pp. 315-321 in Proceedings of the Fourth International Symposium on Multiple-valued Logic, IEEE, Morgantown, 1974.Zbl 0314.02057|MR 51:123 1.11.4.

[57] Yablonskii, S. V., "Functional constructions in a $k$-valued logic," Trudy Math. Inst. Steklov, vol. 51 (1958), pp. 5-142 (in Russian). English translation in: Office of Technology Services, U.S. Department of Commerce, Washington, D.C., 1960.

The editors regret to report the death of Professor Rychkov since the completion of this paper. Professor Anshakov's affiliation appears below.

Faculty of Theoretical \& Applied Linguistics Russian State University for the Humanities

Miusskaya pl. 6

Moscow 125267

Russia

email: anshakov@infao.rggu.msk.su 